\title{
Shot- and angle-domain wave-equation traveltime inversion of reflection data: Theory
}

\author{
Sanzong Zhang ${ }^{1}$, Yi Luo ${ }^{2}$, and Gerard Schuster ${ }^{1}$
}

\begin{abstract}
The main difficulty with iterative waveform inversion is that it tends to get stuck in local minima associated with the waveform misfit function. To mitigate this problem and avoid the need to fit amplitudes in the data, we have developed a wave-equation method that inverts the traveltimes of reflection events, and so it is less prone to the local minima problem. Instead of a waveform misfit function, the penalty function was a crosscorrelation of the downgoing direct wave and the upgoing reflection wave at the trial image point. The time lag, which maximized the crosscorrelation amplitude, represented the reflection-traveltime residual (RTR) that was back projected along the reflection wavepath to update the velocity. Shot- and angle-domain crosscorrelation functions were introduced to estimate the RTR by semblance analysis and scanning. In theory, only the traveltime information was inverted and there was no need to precisely fit the amplitudes or assume a high-frequency approximation. Results with synthetic data and field records revealed the benefits and limitations of wave-equation reflection traveltime inversion.
\end{abstract}

\section{INTRODUCTION}

Full-waveform inversion (FWI) iteratively seeks the velocity model that minimizes a data-domain misfit function, in which the velocity model is updated by the zero-lag temporal correlation between the forward-modeled wavefield and the back-propagated residuals (Tarantola, 1986, 1987; Mora, 1987, 1989; Luo and Schuster, 1991a, 1991b; Crase et al., 1992; Woodward, 1992; Zhou et al., 1995; Pratt, et al., 1998; Virieux and Operto, 2009). A finely detailed velocity model can be inverted if the initial velocity model is close to the true velocity model. The problem with FWI is that its objective function is a nonquadratic function and can be highly oscillatory with respect to velocity perturbations. Therefore, a gradient-based optimization method tends to get stuck in a local minimum if the initial velocity model is far from the true velocity model. To mitigate these difficulties and achieve an accurate solution, FWI often requires a multiscale strategy (Bunks et al., 1995; Sirgue and Pratt, 2004) applied to low-frequency data and densely sampled traces with long offsets in the source-receiver spacings (Plessix et al., 2010). Another problem with FWI is that the physics of wave propagation is only partly accounted for in the forward modeling, so amplitudes cannot be precisely reproduced. Therefore, an inversion method might best restrict itself to exclusively explaining the kinematics of low-frequency wave propagation at the early iterations, and later iterations attempt to explain the amplitudes. An anisotropic model is often required to correctly model the kinematics of all the waves (Plessix and Rynja, 2010).

Reflection-based FWI aims to invert for the long-wavelength components of the velocity model by separating out the tomography term in the conventional FWI kernel (Xu et al., 2011; Zhou et al., 2012; Wang et al., 2013), whereas the velocity update of conventional FWI is dominated by the high-wavenumber migration term in the absence of low-frequency and long-offset data (Tang et al., 2013). Therefore, a hybrid FWI scheme is often used to alternatively update the long and short wavelengths of the model using separate tomography and migration gradients at each iteration. Similar to FWI, reflection-based FWI can still suffer from the cycleskipping problem and cannot fully remedy the problem of incorrect modeling of amplitudes.

A more robust alternative to FWI is wave-equation migration velocity analysis (WEMVA), which is an image-domain method that inverts for the velocity model from the migration images. The goal is to find the velocity model that minimizes the L2 norm of image perturbations so that migrated reflections in the commonimage gathers (CIGs) are flattened or focused in the angle domain

\footnotetext{
Manuscript received by the Editor 16 April 2014; revised manuscript received 1 January 2015; published online 26 May 2015.

${ }^{1}$ Center for Subsurface Imaging and Fluid Modeling (CSIM), King Abdullah University of Science and Technology (KAUST), Thuwal, Saudi Arabia. E-mail: sanzong.zhang@kaust.edu.sa; gerard.schuster@kaust.edu.sa.

${ }^{2}$ Saudi Aramco, Exploration and Petroleum Engineering Center — Advanced Research Center, Dhahran, Saudi Arabia. E-mail: yi.luo@aramco.com.

(C) 2015 Society of Exploration Geophysicists. All rights reserved.
} 
or the subsurface-offset domain. Several WEMVA methods have been proposed to extract velocity information from the migration images. One of them is differential semblance optimization (DSO) (Symes and Kern, 1994; Shen and Calandra, 2005; Shen and Symes, 2008), which uses the first derivative of the migration image with respect to the reflection angle to estimate the image perturbation. It also uses the subsurface offset as a weight to attenuate the energy at far subsurface offsets and focus the image energy at nearsubsurface offsets (Shen et al., 2003; Yang et al., 2013). However, this method suffers from defocusing with subsurface offset-domain CIGs in complex regions with poor illumination even if the velocity model is accurate (Yang et al., 2013). Another method is proposed by Sava and Biondi (2004a), who use residual Stolt migration to construct image perturbations. This approach requires picking a parameter that represents the ratio of the original and modified velocities at every image point; thus, it can require significant human intervention with noisy data (Sava and Biondi, 2004b). Almo$\min$ (2011) uses the depth lag to measure the image perturbation based on the crosscorrelation of the observed image with a reference image in the angle gather. Because the true image depth is difficult to determine in practice, it can be unduly biased by the reference image (Almomin, 2011). The merit of this method is that it is less sensitive to the amplitude of the observed data with minimal cycle-skipping problems. In a related method, Zhang and Biondi (2013) estimate the velocity model by maximizing the angle-stack power of angledomain CIGs. This method relates the image gather flatness to an intermediate moveout parameter. The image perturbation is constructed from this moveout parameter based on the fact that the moveout parameter describes the kinematic change in angle-domain CIGs due to velocity perturbations. However, this approach uses a single parabolic parameter to describe moveouts, which can limit the resolution of fine details in a complex model (Zhang and Biondi, 2013).

In addition to the space-lag methods, WEMVA also inverts the time lag for the velocity model. Yang and Sava (2011) convert the time lags to image perturbations by a linear approximation. The

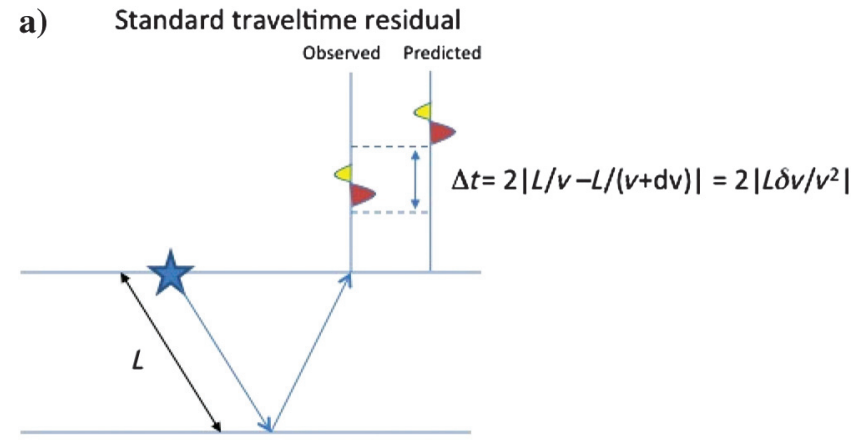

b) Redatumed traveltime residual

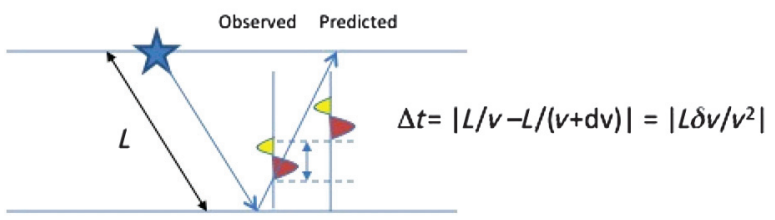

Figure 1. The RTR is computed (a) at the recording plane and (b) at the reflector. The predicted and observed arrivals arrive more closely to one another at the reflector than at the recording plane. conversion from the time lag to the image perturbation may not be valid with large time lags, and a complex-velocity model can spoil accurate picking of the time lag in the time-shift extended image (Wang et al., 2009). To remove the small time-lag approximation, Yang and Sava (2013) focus the migration image at the zero-time lag, and so they directly minimize the time lag similar to wave-equation traveltime inversion (Luo and Schuster, 1991a).

To exploit the strengths and avoid the weaknesses of ray-based traveltime inversion and FWI, wave-equation traveltime inversion was developed to invert the velocity model from transmission traveltimes (Luo and Schuster, 1991a, 1991b; Zhou et al., 1995). This method inverts the time lag that maximizes the crosscorrelation between the observed and calculated transmitted arrivals in crosswell data. Only the traveltime information is inverted, so there is no need to fit the amplitudes or assume a high-frequency approximation. Another benefit is that only the transmission arrivals are inverted, so the method enjoys a robust convergence rate. A shortcoming of Wave-equation reflection traveltime inversion (WT) is that it only inverts reflection traveltimes if specified reflections are isolated in the data. Unlike refraction and turning waves with modest sourcereceiver offsets, reflection traveltimes can provide significant information about the deeper parts of the subsurface. To eliminate the specification of reflection events in the data, Zhang et al. (2011) present a modified extension of WT as wave-equation reflection traveltime inversion. Because the residual moveout analysis in the angle-domain CIGs provides a robust estimate of the depth residual, Zhang et al. (2012) convert the depth residuals to the time lags and then invert them by the WT method. Al-Saleh and Jiao (2012) use a similar approach with the one-way wave equation. However, time-lag picking can still be a problem for complex velocity models, even with the approach used by Luo and Hale (2013) with warping of migration images (Ma and Hale, 2013). Unless otherwise noted, the WT method applied to reflection traveltimes will also be denoted as WT.

Our proposed WT method for reflection traveltimes aims to fit the observed and predicted reflections at the reflector itself, not at the recording plane. In this way, the predicted and observed arrivals arrive more closely to one another, even for large velocity errors as shown in Figure 1. This reduces, but does not eliminate, the tendency for cycle skipping. The key step is to redatum the reflection arrivals to the trial reflection depth and perform the correlation at this reflection level. Compared with ray-based MVA, the proposed method does not require a high-frequency approximation, produces tomograms with moderately high resolution, and the conversion from the depth residual to the time residual is not required.

We next present the basic theory of shot- and angle-domain wave-equation traveltime inversion and further discuss its advantages and limitations. Then, we describe the key step for estimating traveltime residuals in shot-domain and angle-domain image gathers. Finally, we present the workflow in which measured residuals are used to update the velocity model. A companion paper (Zhang et al., 2015) shows how the method performs on synthetic and real data sets.

\section{THEORY}

This section presents the derivation of the shot- and angledomain WT methods, and compares the characteristics of WT and FWI. The detailed derivation can be found in Appendix A. 


\section{Shot- and angle-domain crosscorrelation function}

The crosscorrelation between the forward-modeled wavefield $p_{s}\left(\mathbf{x}, t \mid \mathbf{x}_{s}\right)$ initiated at the source location $\mathbf{x}_{s}$ and the backwardpropagated wavefield $p_{g}\left(\mathbf{x}, t \mid \mathbf{x}_{s}\right)$ at a trial image point $\mathbf{x}$ is defined as

$$
f\left(\mathbf{x}, \mathbf{x}_{s}, \tau\right)=\int p_{s}\left(\mathbf{x}, t+\tau \mid \mathbf{x}_{s}\right) p_{g}\left(\mathbf{x}, t \mid \mathbf{x}_{s}\right) \mathrm{d} t,
$$

where $\tau\left(\mathbf{x}, \mathbf{x}_{s}\right)$ is the time lag of the crosscorrelation function $f\left(\mathbf{x}, \mathbf{x}_{s}, \tau\right)$. We also denote $p_{s}\left(\mathbf{x}, t \mid \mathbf{x}_{s}\right)$ as the wavefield of the redatumed source and $p_{g}\left(\mathbf{x}, t \mid \mathbf{x}_{s}\right)$ as the redatumed reflection data.

The crosscorrelation function in equation 1 for a common shot gather is denoted as a shot-domain crosscorrelation function. However, the crosscorrelation function can be expressed as the angle-domain crosscorrelation function defined in the reflection-angle domain, which is converted from the subsurface offset-domain crosscorrelation function by slant stacking (Sava and Fomel, 2003). The angle-domain crosscorrelation function is defined as

$$
\begin{aligned}
f(\mathbf{x}, \theta, \tau)= & \int d \mathbf{x}_{s} \int d h \int p_{s}(x-h, z+h \tan \theta, t \\
& \left.+\tau \mid \mathbf{x}_{s}\right) p_{g}\left(x+h, z+h \tan \theta, t \mid \mathbf{x}_{s}\right) \mathrm{d} t,
\end{aligned}
$$

where $h$ is the subsurface offset and $\theta$ is the reflection angle in the $2 \mathrm{D}$ case. Because this conversion method is valid for $3 \mathrm{D}$ only in the absence of crossline structure dip (Biondi and Symes, 2004) and the computation is very complex in 3D (Fomel, 2004), some alternatives can be used to form the angle-domain crosscorrelation. Other methods can be used to create the angle gathers in 3D if Sava and Fomel's method is too expensive or too difficult to implement in a particular application (Xu et al., 2011).

For a variation of the subsurface offset parameter $h$ within a certain range, $p_{s}(x-h, z+$ $\left.h \tan \theta, t \mid \mathbf{x}_{s}\right)$ represents a local downgoing plane wave and $p_{g}\left(x+h, z+h \tan \theta, t \mid \mathbf{x}_{s}\right)$ denotes a local upgoing plane wave as shown in Figure 2. This is because the coordinates $(x-h, z+$ $h \tan \theta)$ and $(x+h, z+h \tan \theta)$ describe a local plane that depends on the subsurface offset variable $h$. This means that the angle-domain crosscorrelation function is defined in terms of local downgoing and upgoing plane waves. The angle-domain crosscorrelation function should provide a more robust estimate of the reflectiontraveltime residual (RTR) compared with the shot-domain crosscorrelation function.

Figure 3 shows the difference between the angle-domain and shot-domain crosscorrelation functions. The shot-domain crosscorrelation function is defined for only one reflection point of the wavefront as shown in Figure 3b, whereas the angle-domain crosscorrelation function is defined for a series of points on the wavefront with respect to the subsurface offset variable $h$ as shown in Figure 3a. It is obvious that the angledomain crosscorrelation function has a higher

a) signal-to-noise ratio than the shot-domain crosscorrelation function as shown in Figure 3, and so it is a more robust means for estimating the RTR. Another benefit of the angle-domain crosscorrelation function compared to the shot-domain crosscorrelation function is that the angle-domain crosscorrelation can account for multiarrivals with different reflection angles. The shot-domain crosscorrelation function is characterized by several peaks for many multiarrival reflections. Only one of them is picked as a traveltime residual. However, the different pairs of reflections can be separated in the reflection-angle domain, and the traveltime residual for an individual reflection can be estimated by the crosscorrelation of traces (a local plane wave) with different reflection angles. a) Local downgoing plane wave

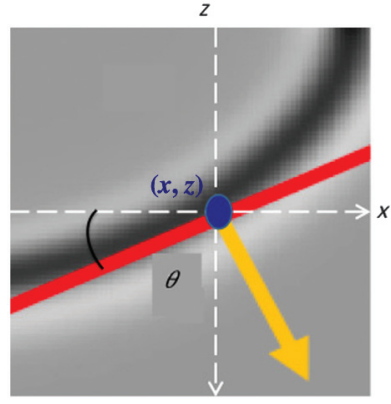

b) Local upgoing plane wave

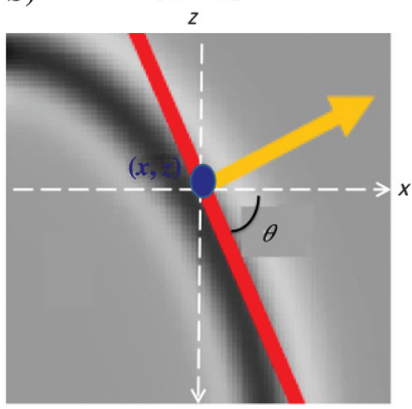

Figure 2. For a small range of $h$ values, the red lines sample the wavefront as (a) a local downgoing plane wave and (b) a local upgoing plane wave. Here, $\theta$ is the complementary angle of the reflection angle. For convenience, we still call $\theta$ the reflection angle in the paper.

Angle-domain crosscorrelation

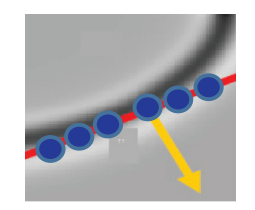

Local downgoing plane wave
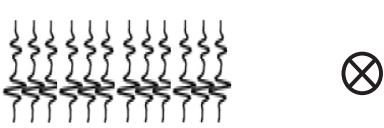

b)

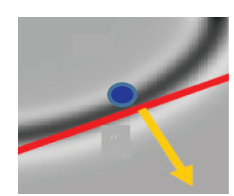

Downgoing wave

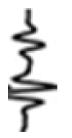

Shot-domain crosscorrelation

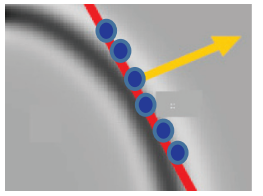

Local upgoing plane wave
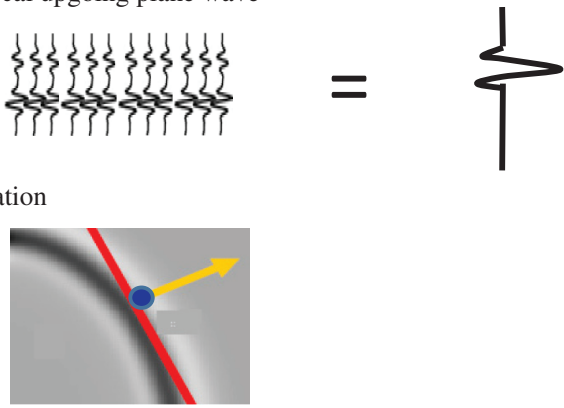

Upgoing wave

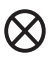

Figure 3. (a) The angle-domain crosscorrelation function is the crosscorrelation between a local downgoing plane wave and a local upgoing plane wave at a series of points on the wavefront. (b) The shot-domain crosscorrelation function is the crosscorrelation between a downgoing wave and an upgoing wave only at one point on the wavefront. 
The proposed WT method attempts to invert for the traveltime residual estimated by the crosscorrelation of the observed and predicted reflections at the reflector itself, not at the recording plane. To demonstrate that the predicted reflections redatumed to the image point are more closely aligned with the actual reflections, we generate synthetic data for the velocity model in Figure 4, where the synthetic data has a peak frequency of $15 \mathrm{~Hz}$. There are 128 sources and 801 receivers evenly distributed on the top surface of the model. The local velocity anomalies create many diffraction events in the observed data that make it difficult to estimate the RTRs at the recording surface for a background velocity model of $2000 \mathrm{~m} / \mathrm{s}$. The migration image and the crosscorrelation functions at the offsets 3.5 and $4.5 \mathrm{~km}$ are shown in Figures 5 and 6 for the background veloc- a)

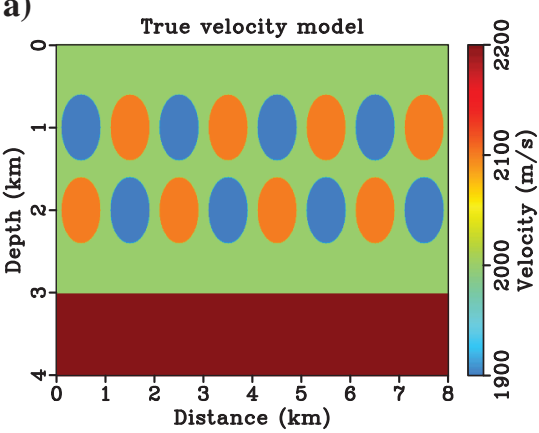

b)

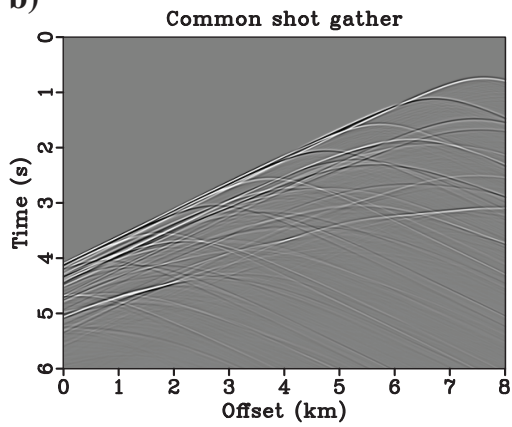

ity model. These examples illustrate that it is feasible to estimate the traveltime residuals by the crosscorrelation of predicted and observed traces at reflectors even for some complex structures and poor starting models.

\section{Misfit function}

The extremum of $f\left(\mathbf{x}, \mathbf{x}_{s}, \tau\right)$ should satisfy

$$
f\left(\mathbf{x}, \mathbf{x}_{s}, \Delta \tau\right)=\max \left\{f\left(\mathbf{x}, \mathbf{x}_{s}, \tau\right) \mid \tau \in[-T, T]\right\},
$$

or

$$
\begin{aligned}
f\left(\mathbf{x}, \mathbf{x}_{s}, \Delta \tau\right) & =\min \left\{f\left(\mathbf{x}, \mathbf{x}_{s}, \tau\right) \mid \tau\right. \\
& \in[-T, T]\},
\end{aligned}
$$

where $T$ is the estimated maximum time lag between the forward-modeled wavefield from the source and the backward-propagated wavefield from the receivers and $\Delta \tau$ is the time lag that maximizes or minimizes the crosscorrelation function. At the trial image point $\mathbf{x}$, this time lag $\Delta \tau$ represents the arrival time delay between the forward- and backward-propagated waves. Here, $\Delta \tau\left(\mathbf{x}_{s}, \mathbf{x}\right)$ is a function dependent on the source and image points. It is also an implicit function of the velocity model (Luo and Schuster, 1991a, 1991b). Note, $\Delta \tau=0$ indicates that the correct velocity model has been found, which generates a downgoing direct wave and upgoing reflection wave arriving at the reflection point at the same time. The derivative of $f\left(\mathbf{x}, \mathbf{x}_{s}, \tau\right)$ with respect to $\tau$ should be zero at $\Delta \tau$ unless its maximum or minimum is at an end point $T$

$$
\begin{aligned}
\dot{f}_{\Delta \tau} & =\left.\frac{\partial f\left(\mathbf{x}, \mathbf{x}_{s}, \tau\right)}{\partial \tau}\right|_{\tau=\Delta \tau} \\
& =\int d t \dot{p}_{s}\left(\mathbf{x}, t+\Delta \tau \mid \mathbf{x}_{s}\right) p_{g}\left(\mathbf{x}, t \mid x_{s}\right)=0
\end{aligned}
$$
of the forward-propagated wave. Equation 5 is called the connective function, which is an implicit function connecting the time lag with the velocity model (Luo and Schuster, 1991a, 1991b). The inverse problem is defined as finding a velocity model that minimizes the following misfit

Here, $\mathbf{x}$ is the trial image position, $\mathbf{x}_{s}$ is the source position, and $\Delta \tau\left(\mathbf{x}_{s}, \mathbf{x}\right)$ denotes the traveltime delay at the trial image point $\mathbf{x}$ between the forward-

propagated wave from the source $\mathbf{x}_{s}$ and the back-
Figure 5. Shot- and angle-domain crosscorrelation functions at the offset of $3.5 \mathrm{~km}$. Panels ( $\mathrm{a}$ and $\mathrm{b}$ ) are the shot-domain crosscorrelations at depths of 2.4 and $3.5 \mathrm{~km}$. Panels (c and d) are the angle-domain crosscorrelation functions at depths of 2.4 and $3.5 \mathrm{~km}$ or $-T$ :

where $\dot{p}_{s}\left(\mathbf{x}, t \mid \mathbf{x}_{s}\right)$ represents the time derivative function:

$$
S=\frac{1}{2} \sum_{\mathbf{x}_{s}} \sum_{\mathbf{x}}\left[\Delta \tau\left(\mathbf{x}_{s}, \mathbf{x}\right)\right]^{2} .
$$
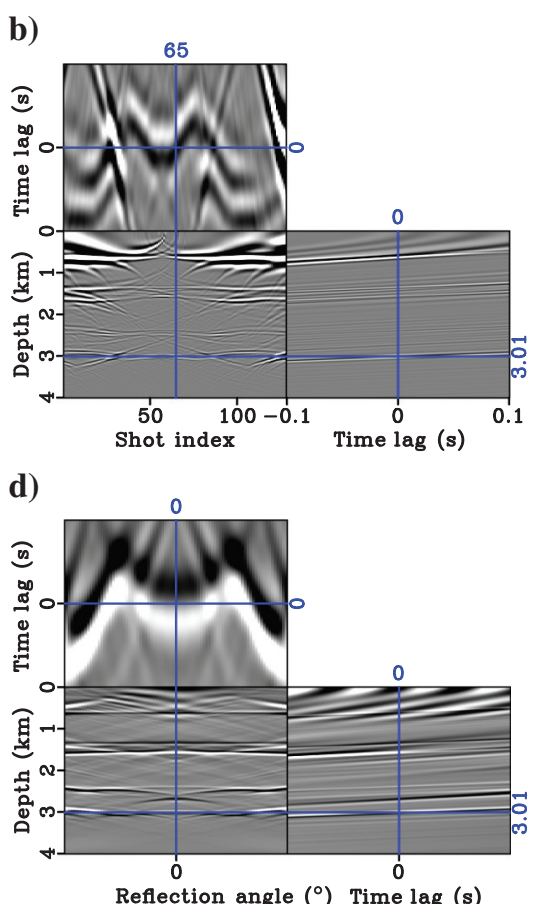
ward-propagated wave from the receivers at $\mathbf{x}_{g}$. It is also the time lag or the RTR defined in equations 1 . In practice, for transmission-traveltime inversion, these traveltimes can be found easily by an automated method such as crosscorrelating the observed seismogram with the calculated seismogram (Luo and Schuster, 1991a, 1991b). The time lag that maximizes the crosscorrelation function indicates the traveltime difference between the observed and calculated reflection arrivals. However, this method cannot be directly applied to the reflection data due to the crosstalk among many other reflection events.

For the angle-domain crosscorrelation function, the extremum of $f(\mathbf{x}, \theta, \tau)$ should satisfy

$$
f(\mathbf{x}, \theta, \Delta \tau)=\max \{f(\mathbf{x}, \theta, \tau) \mid \tau \in[-T, T]\},
$$

or

$$
f(\mathbf{x}, \theta, \Delta \tau)=\min \{f(\mathbf{x}, \theta, \tau) \mid \tau \in[-T, T]\},
$$

where $\Delta \tau$ is the stationary time lag that maximizes or minimizes the correlation function for the reflection angle $\theta$. The derivative of $f(\mathbf{x}, \theta, \tau)$ with respect to $\tau$ should be zero at $\Delta \tau$ unless its maximum or minimum is at an end point $T$ or $-T$ :

$$
\dot{f}_{\Delta \tau}=\left.\frac{\partial f(\mathbf{x}, \theta, \tau)}{\partial \tau}\right|_{\tau=\Delta \tau}=0
$$

where the associated misfit function is defined in the angle domain as

$$
S=\frac{1}{2} \sum_{\mathbf{x}} \sum_{\theta}[\Delta \tau(\mathbf{x}, \theta)]^{2} .
$$

Here, $\Delta \tau(\mathbf{x}, \theta)$ denotes the traveltime delay at the trial image point $\mathbf{x}$ between the forward- and backward-propagated local plane waves associated with the reflection angle $\theta$.

The optimal estimate of the velocity model $c\left(\mathbf{x}^{\prime}\right)$ minimizes the sum of the squared RTRs in equations 6 or 10 . Here, $\mathbf{x}^{\prime}$ represents any location in the velocity model. For simplicity, a steepest descent optimization method is used to describe the iterative optimization, with the understanding that a preconditioned conjugate gradient method is used in practice. To update the velocity model, the steepest descent method gives

$$
c_{k+1}\left(\mathbf{x}^{\prime}\right)=c_{k}\left(\mathbf{x}^{\prime}\right)+\alpha_{k}\left(\mathbf{x}^{\prime}\right) \cdot \gamma_{k}\left(\mathbf{x}^{\prime}\right),
$$

where $\gamma_{k}\left(\mathbf{x}^{\prime}\right)$ is the steepest descent direction for the misfit function $S, \alpha_{k}$ is the step length, and $k$ denotes the $k$ th iteration.

\section{Gradient function}

Under the Born approximation, the misfit gradient of the objective function 6 can be written as

$$
\gamma\left(\mathbf{x}^{\prime}\right)=\gamma_{1}\left(\mathbf{x}^{\prime}\right)+\gamma_{2}\left(\mathbf{x}^{\prime}\right),
$$

where

$$
\begin{aligned}
& \gamma_{1}\left(\mathbf{x}^{\prime}\right)=\frac{2}{c\left(\mathbf{x}^{\prime}\right)^{3}} \sum_{\mathbf{x}_{s}} \sum_{\mathbf{x}} \\
& \times \int \overbrace{\left[\int \frac{\Delta \tau}{E} \dot{p}_{g}\left(\mathbf{x}_{g}, t \mid \mathbf{x}_{s}\right) * g\left(\mathbf{x}^{\prime},-t \mid \mathbf{x}_{g}, 0\right) d \mathbf{x}_{g}\right]}^{\text {back propagation of the observed data }}
\end{aligned}
$$

forward propagation of the redatumed source

$$
\overbrace{\left[\ddot{p}_{s}\left(\mathbf{x}, t+\Delta \tau \mid \mathbf{x}_{s}\right) * g\left(\mathbf{x}^{\prime}, t \mid \mathbf{x}, 0\right)\right]}
$$

and

$$
\begin{gathered}
\gamma_{2}\left(\mathbf{x}^{\prime}\right)=\frac{2}{c\left(\mathbf{x}^{\prime}\right)^{3}} \sum_{\mathbf{x}_{s}} \sum_{\mathbf{x}} \\
\times \int \overbrace{\left[\begin{array}{c}
\text { back propagation of the redatumed data } \\
\text { forward propagation of the source }
\end{array}\right.}^{\overbrace{\left[\dot{p}_{s}\left(\mathbf{x}^{\prime}, t+\Delta \tau \mid \mathbf{x}_{s}\right)\right]}} \mathrm{d} t,
\end{gathered}
$$

which are derived in Appendix A. The WT gradient in equation 12 consists of two terms associated with two virtual-transmission experiments. In the virtual experiment for $\gamma_{1}\left(\mathbf{x}^{\prime}\right)$, the source is redatumed to the image point at $\mathbf{x}$ and the geophones are still on the recording surface at $\mathbf{x}_{g}$ as shown in Figure 7a. In the other virtual experiment for $\gamma_{2}\left(\mathbf{x}^{\prime}\right)$, the receivers are redatumed to the image point at $\mathbf{x}$ and the source is on the recording surface at $\mathbf{x}_{s}$ as shown

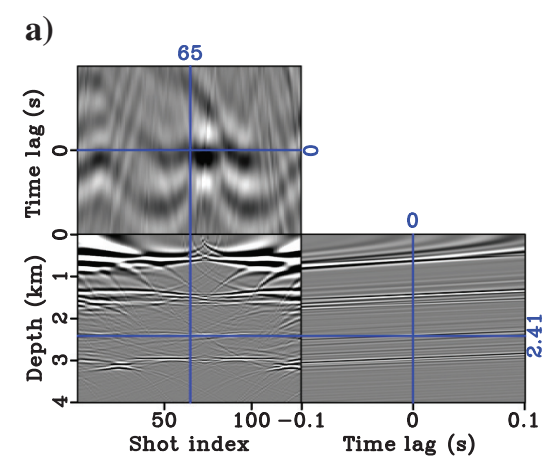

c)

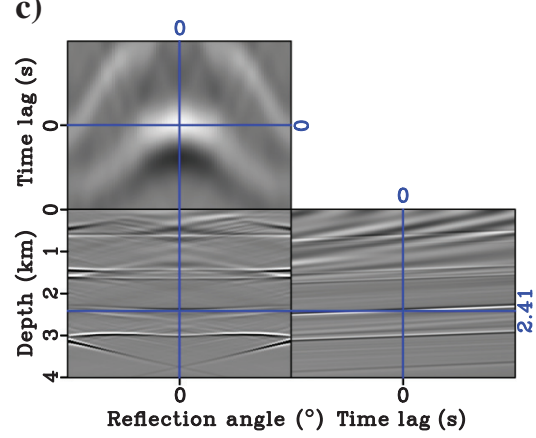

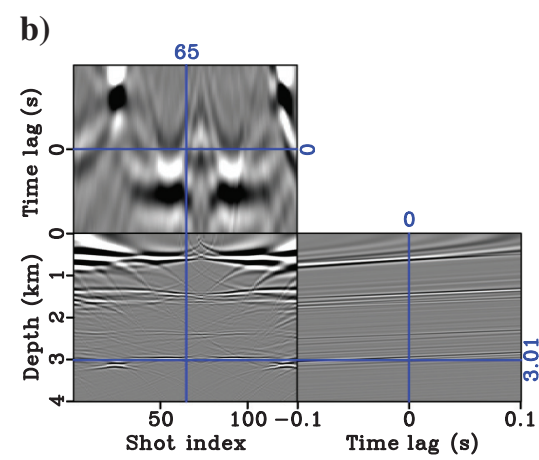

d)

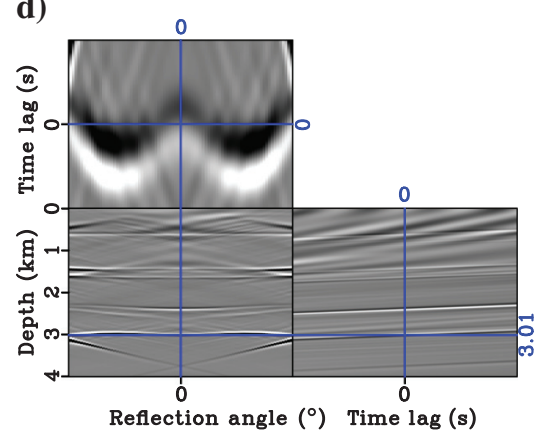

Figure 6. Same as the previous figure, except the offset is $4.5 \mathrm{~km}$. 
in Figure 7b. The time lag is derived from the crosscorrelation between the traces associated with the redatumed source, and the redatumed data shown in Figure $8 \mathrm{a}$. The velocity model is updated by

a)

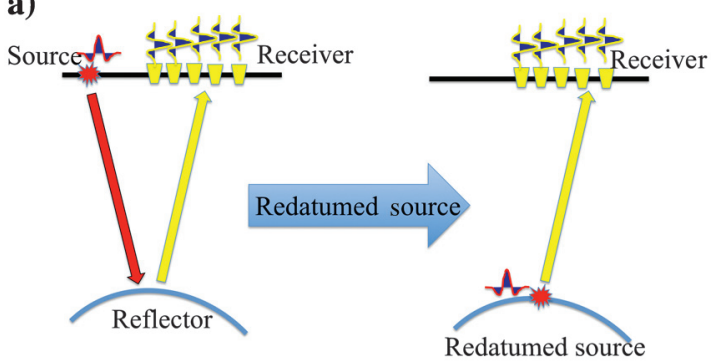

b)

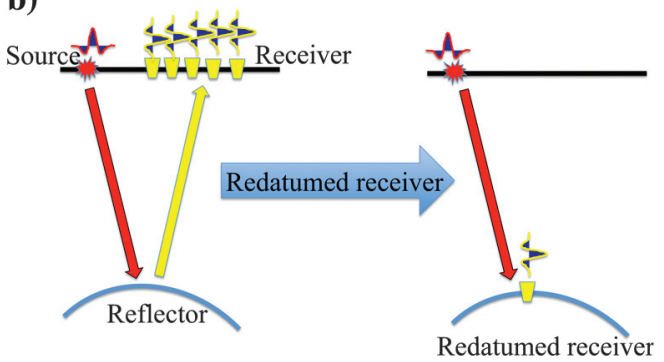

Figure 7. Ray diagrams illustrate (a) the forward extrapolation of the source field and (b) the backward extrapolation of the geophone field.

a) Redatumed source Redatumed receiver Crosscorrelation
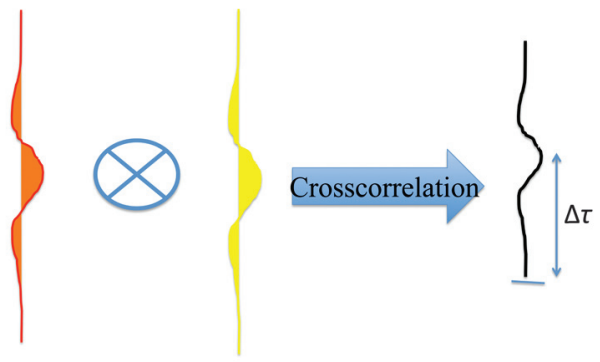

b)

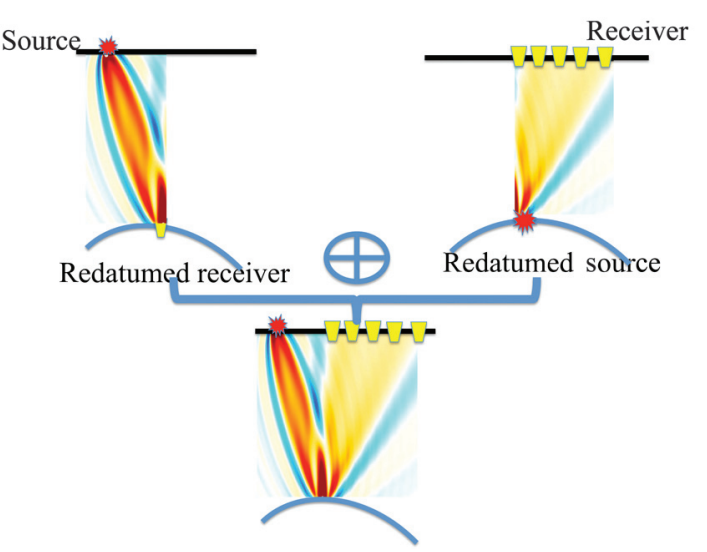

Figure 8. Ray diagrams illustrate (a) the crosscorrelation of the downgoing direct and the upgoing reflection wavefields and (b) that the misfit gradient is proportional to the wavepath function weighted by $\Delta \tau$ for each source-receiver. smearing the time lag calculated with the virtual traces at the image points along the wavepaths between the source and the image points, and the image points and the geophones are shown in Figure 8b. The key benefit of these redatuming steps is that the events in the redatumed traces are not as weak or complex as those recorded on the surface, so the correlation of the reflection field at the reflector with the source field has fewer artifacts.

The misfit gradient of the angle-domain objective function in equation 10 is

$$
\begin{gathered}
\gamma\left(\mathbf{x}^{\prime}\right)=\frac{2}{c(\mathbf{x} \prime)^{3}} \sum_{\theta} \sum_{\mathbf{x}} \frac{\Delta \tau(\mathbf{x}, \theta)}{E} \int d \mathbf{x}_{s} \int d h \int \dot{p}_{g}\left(\mathbf{x}^{\prime}, t \mid \mathbf{x}_{s}\right) \\
{\left[\ddot{p}_{s}\left(\mathbf{x}-h, z+h \tan \theta, t+\Delta \tau \mid \mathbf{x}_{s}\right) * g\left(\mathbf{x}^{\prime}, t \mid \mathbf{x}+h, z+h \tan \theta, 0\right)\right] \mathrm{d} t} \\
+\frac{2}{c\left(\mathbf{x}^{\prime}\right)^{3}} \sum_{\theta} \sum_{\mathbf{x}} \frac{\Delta \tau(\mathbf{x}, \theta)}{E} \int d \mathbf{x}_{s} \int d h \int \dot{p}_{s}\left(\mathbf{x}^{\prime}, t+\Delta \tau \mid \mathbf{x}_{s}\right) \\
{\left[\ddot{p}_{g}\left(\mathbf{x}+h, z+h \tan \theta, t \mid \mathbf{x}_{s}\right) * g\left(\mathbf{x}^{\prime},-t \mid \mathbf{x}-h, z+h \tan \theta, 0\right)\right] \mathrm{d} t .}
\end{gathered}
$$

Equation 15 has a physical interpretation similar to that of equations 13 and 14. The difference is that the backward-propagated wave is a local plane wave with a time shift associated with a certain reflection angle.

A numerical example is now used to compare the gradient function in the shot domain with that in the angle domain. Figure 9a shows the true velocity model, which is a two-layered model. The first layer contains an anomaly with a high-velocity perturbation. A common shot gather is displayed in Figure 9b, in which there are 128 sources and 801 receivers distributed evenly on the top surface of the model. Figure 10 shows the shot- and angle-domain crosscorrelation traces at offsets of 2 and $4 \mathrm{~km}$. The traveltime residual can be picked from these crosscorrelograms, in which the gradients of the objective functions using the constant velocity of $2000 \mathrm{~m} / \mathrm{s}$ are shown in Figure 11. Figure 11a and 11c shows the sum of the gradient functions for all points on the reflector, whereas Figure 11b and $11 \mathrm{~d}$ shows the gradient functions for a single point on the reflector. This example shows that shot- and angle-domain gradient functions can provide the correct velocity update.

\section{Relationship of WT inversion with full-waveform inversion}

We show that the shot-domain gradient function of the WT method approximates that of FWI if the starting velocity model is close to the true velocity model. Although the starting velocity model is far from the true velocity model, FWI has a cycle-skipping problem because the calculated arrivals can be significantly time shifted with respect to the observed reflections. However, the WT method can mitigate this type of cycle skipping with updating the velocity model by smearing the data residual along the reflection wavepath weighted by the RTR. Here, the starting velocity model is assumed to be close to the true velocity model. If $p\left(\mathbf{x}, t \mid \mathbf{x}_{s}\right)$ is the calculated wavefield for the current starting velocity model, then the calculated arrival $p\left(\mathbf{x}, t \mid \mathbf{x}_{s}\right)$ differs by only a minor time shift $\Delta \tau$ from the arrivals $p_{s}\left(\mathbf{x}, t \mid \mathbf{x}_{s}\right)$ and $p_{g}\left(\mathbf{x}, t \mid \mathbf{x}_{s}\right)$, 


$$
p\left(\mathbf{x}, t \mid \mathbf{x}_{s}\right) \approx p_{s}\left(\mathbf{x}, t+\Delta \tau \mid \mathbf{x}_{s}\right) \approx p_{g}\left(\mathbf{x}, t \mid \mathbf{x}_{s}\right)
$$

The wavefield perturbation $\Delta p\left(\mathbf{x}, t \mid \mathbf{x}_{s}\right)$ between the calculated data and the observed data is represented by

$$
\Delta p\left(\mathbf{x}, t \mid \mathbf{x}_{s}\right) \approx \Delta \tau \dot{p_{g}}\left(\mathbf{x}, t \mid \mathbf{x}_{s}\right) .
$$

Substituting equations 16 and 17 into 13 and 14, we obtain

$$
\begin{aligned}
& \gamma\left(\mathbf{x}^{\prime}\right)=\frac{2}{c\left(\mathbf{x}^{\prime}\right)^{3}} \sum_{\mathbf{x}_{s}} \sum_{\mathbf{x}} \\
& \times \int \overbrace{\left[\frac{1}{E} \Delta \dot{p}\left(\mathbf{x}, t \mid \mathbf{x}_{s}\right) * g\left(\mathbf{x}^{\prime},-t \mid \mathbf{x}, 0\right)\right]}^{\text {back propagation of the redatumed data }} \\
& \text { forward propagation of the source } \mathrm{d} t+ \\
& \overbrace{\left[\dot{p}\left(\mathbf{x}^{\prime}, t \mid \mathbf{x}_{s}\right)\right]}
\end{aligned}
$$$$
\frac{2}{c\left(\mathbf{x}^{\prime}\right)^{3}} \sum_{\mathbf{x}_{s}} \sum_{\mathbf{x}} \int \overbrace{\left[\int \frac{1}{E} \Delta p\left(\mathbf{x}_{g}, t \mid \mathbf{x}_{s}\right) * g\left(\mathbf{x}^{\prime},-t \mid \mathbf{x}_{g}, 0\right) d \mathbf{x}_{g}\right]}^{\text {back propagation of the observed data }}
$$

forward propagation of the redatumed source

$$
\overbrace{\left[\ddot{p}\left(\mathbf{x}, t \mid \mathbf{x}_{s}\right) * g\left(\mathbf{x}^{\prime}, t \mid \mathbf{x}, 0\right)\right]}
$$

Equation 18 is the tomographic components in the misfit gradient for FWI, which updates the smooth components of the velocity model (Mora, 1989; Zhou et al., 2012; Tang et al., 2013; Wang et al., 2013; Xu et al., 2013). This derivation demonstrates that if the starting velocity model is close to the true velocity model, then the WT and FWI gradient functions are similar to one another. The difference is that when the starting velocity model is far from the true velocity model, the assumption of $\Delta \tau<T / 2$ is violated and FWI can suffer from cycle skipping. In contrast, WT mitigates cycle skipping by using the inner product between the source wavefield $\dot{p_{s}}\left(\mathbf{x}^{\prime}, t+\Delta \tau \mid \mathbf{x}_{s}\right)$, which is shifted by $\Delta \tau$, and the weighted receiver wavefield $\Delta \tau \ddot{p}_{g}\left(\mathbf{x}, t \mid \mathbf{x}_{s}\right) * g\left(\mathbf{x}^{\prime},-t \mid \mathbf{x}, 0\right)$. Here, the weight is the traveltime residual $\Delta \tau$ to ensure the correct trend of the velocity update, and the source wavefield is shifted by $\Delta \tau$ to avoid getting out of phase with the receiver wavefield.

The crosscorrelation of two traces can still have a cycle -skipping problem, but this problem is reduced in WT because the back propagated predicted and observed reflections arrive at nearly the same time compared to their arrivals at the recording surface. After the traveltime time residual is picked from the crosscorrelation, we use it as a time delay to align the source and residual wavefields in phase. Therefore, the zero-lag crosscorrelation of the source wavefield and its adjoint wavefield weighted by the time shift produces long-wavelength updates without cycle skipping. The gradient functions for FWI and WT are shown in Figures 11 and 12, in which the FWI gradient did not provide a correct velocity update due to cycle skipping. The comparison illustrates that WT can provide a more accurate velocity update than the standard FWI.

\section{NUMERICAL EXAMPLES}

\section{Calculation of reflection-traveltime residuals}

An efficient and accurate estimate of the RTR $\Delta \tau$ is a crucial step for WT. This RTR is the time lag, which yields the maximum amplitude of the crosscorrelation function. We can pick $\Delta \tau$ directly from the crosscorrelation function, but this is too labor intensive. Our preferred method is to automate the picking by fitting a parabola to the RTR curve in the crosscorrelation function. The procedure for estimating the RTR involves three steps: (1) calculate the shot- or angle-domain crosscorrelation function, (2) determine the location of the reflection point from the CIGs, and (3) conduct semblance analysis to find a parabolic function to fit the reflection-traveltime curve. The parabolic equation used for semblance analysis is

$$
\Delta \tau=\alpha(\Delta x)^{2},
$$

where $\alpha$ is the curvature of the parabolic curve and $\Delta x$ is the horizontal distance between the locations of the reflection and source points.

A layered model example is now used to describe the estimation of the shot-domain RTR. Figure 13a depicts the reflectivity model with the constant velocity of $2000 \mathrm{~m} / \mathrm{s}$. The five reflection events in Figure $13 \mathrm{~b}$ are generated by Born modeling with a $20-\mathrm{Hz}$ Ricker wavelet. Figure 14 illustrates the estimate of the shot-domain RTR, in which reverse time migration (RTM) is used to calculate the crosscorrelation function $f\left(z, x_{0}, x_{s}, \Delta \tau\right)$ in Figure $14 \mathrm{a}$ at $\mathbf{x}_{0}$. The migration velocity is the constant-velocity model of $1600 \mathrm{~m} / \mathrm{s}$, which is $20 \%$ less than the true velocity of $2000 \mathrm{~m} / \mathrm{s}$. The approximate depth of the reflector $z_{0}$ is chosen from the shot-domain CIGs, which is actually the zero-time-lag crosscorrelation function $f\left(z_{0}, x_{0}, x_{s}, \Delta \tau=0\right)$. Semblance analysis is then applied to the crosscorrelation function $f\left(z_{0}, x_{0}, x_{s}, \Delta \tau\right)$ at the chosen location. We pick the highest energy curve in the semblance spectrum and find the corresponding curvature parameter $\alpha$ in Figure 14b. Inserting this parameter into equation 19 , we can calculate the RTR analytically. We also calculate the shot-domain crosscorrelation function and semblance spectrum from the true velocity model as shown in Figure 14c and 14d. The energy cluster at the zero-time
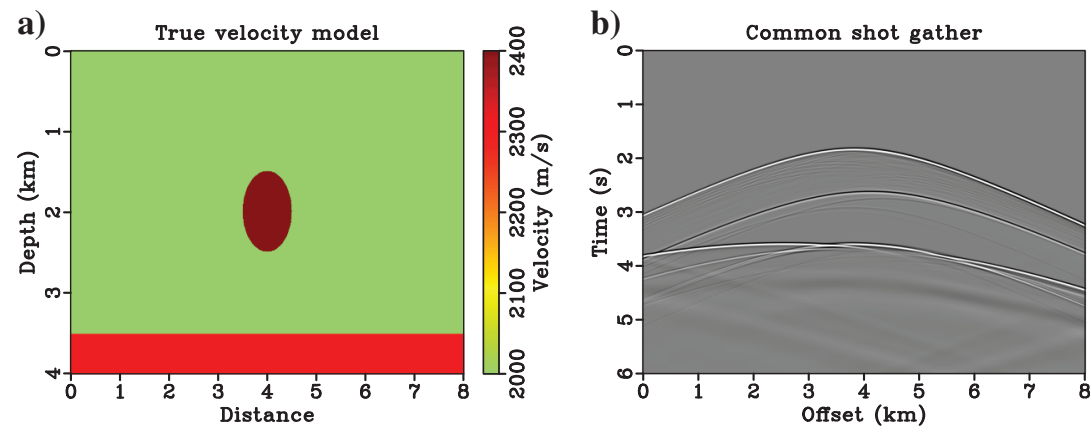

Figure 9. (a) True velocity model, in which the background velocity model is the constant velocity of $2000 \mathrm{~m} / \mathrm{s}$ with a high-velocity perturbation. (b) A common shot gather with peak frequency of $15 \mathrm{~Hz}$. There are 128 sources and 801 receivers evenly distributed on the top of the model. 
lag exhibits zero curvature in the semblance spectrum, which means that the true velocity model collapses the RTR at the zero-time-lag parameter $\Delta \tau=0$ in the crosscorrelation function. a)

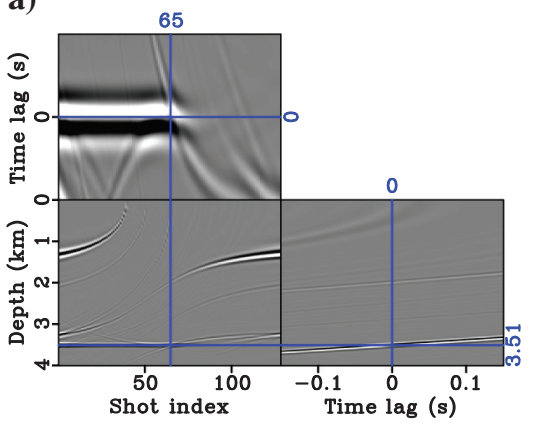

c)

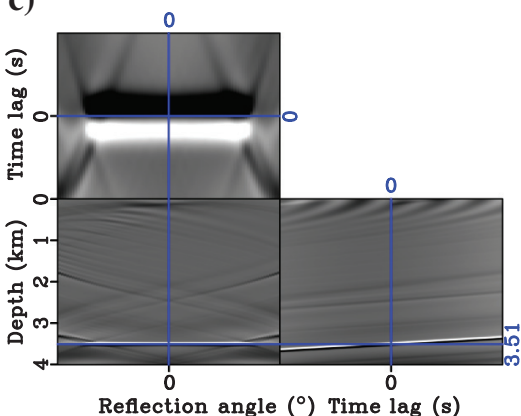

b)

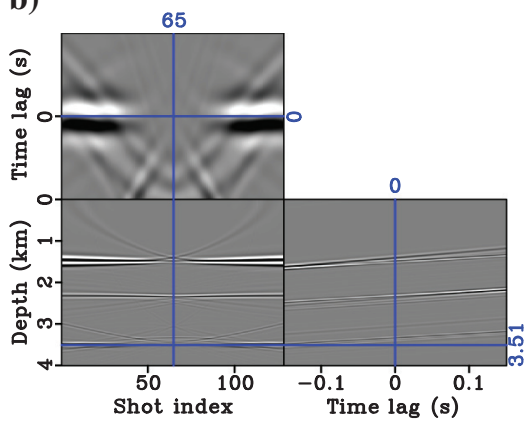

d)

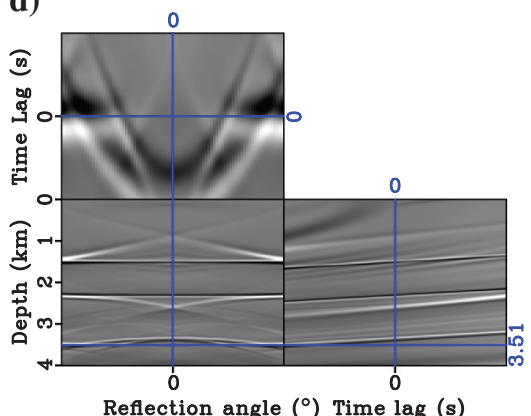

Figure 10. The same as Figure 6, except the observed data are computed for the Figure 9 model. The background velocity model is $2000 \mathrm{~m} / \mathrm{s}$. The offset is $2 \mathrm{~km}$ for panels (a and b) and $4 \mathrm{~km}$ for panels (c and d).
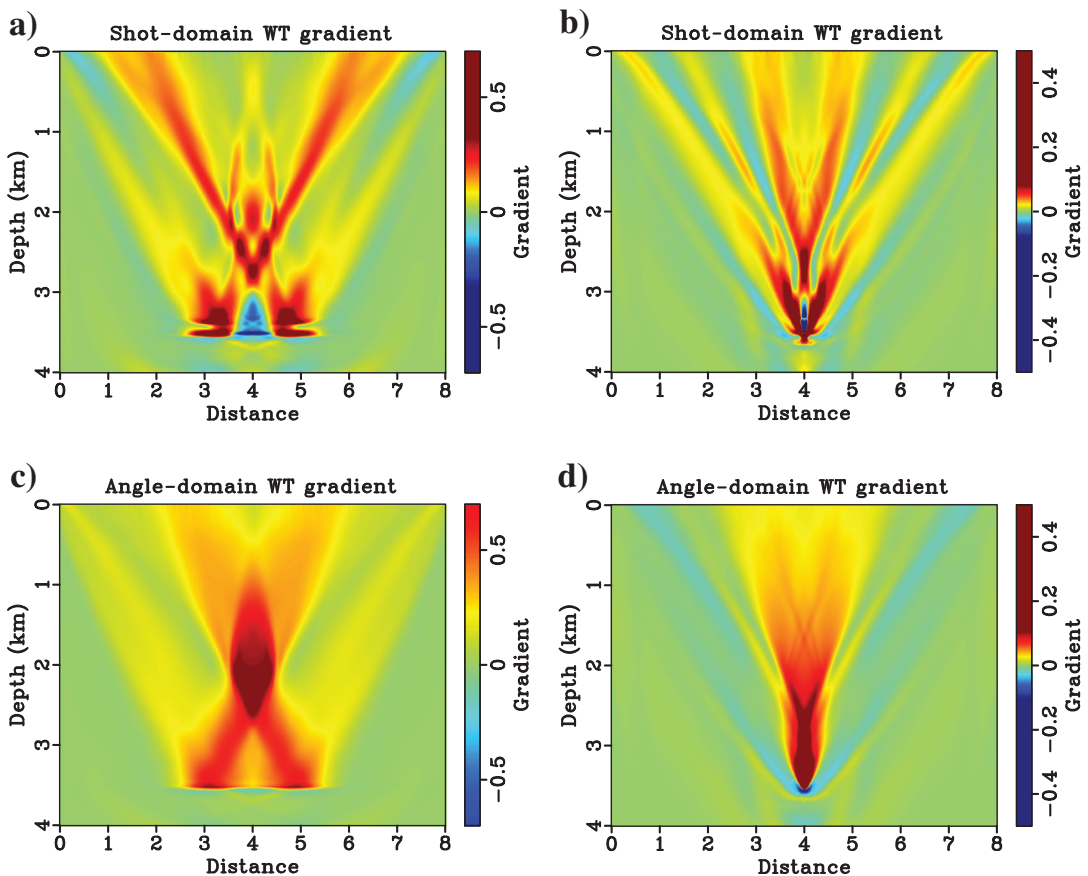

Figure 11. Shot- and angle-domain gradient functions for the constant background velocity model $2000 \mathrm{~m} / \mathrm{s}$. Panels (a and b) depict the shot-domain gradient functions for a single reflection point on the reflector. Panels (c and d) show the angle-domain gradient functions calculated for a single point on the reflector.
The same procedure is used to calculate the angle-domain RTR. The only difference is the equation used to fit the angle-domain RTR curve:

$$
\Delta \tau=\alpha(\tan \theta)^{2},
$$

where $\alpha$ is the curvature parameter and $\theta$ is the reflection angle. Figure $15 \mathrm{a}$, and $15 \mathrm{~d}$ illustrates the estimate of the angle-domain RTR. The angle-domain crosscorrelation functions shown in Figure $15 \mathrm{a}$ and $15 \mathrm{c}$ are computed from the subsurface offset-domain crosscorrelation functions by a slant-stack procedure. The value of $\alpha$ is picked from the semblance spectrum in Figure 15b and $15 \mathrm{~d}$ to calculate the angle-domain RTR with equation 20.

Moveout equations 19 and 20 for the traveltime residual are referenced to the moveout equations of the depth residual (Biondi and Symes, 2004). The curvature is the only parameter to determine, and it is only valid in cases of longwavelength velocity errors. The moveout equation with multiparameters is required to extract the traveltime residuals in the case of complex velocity errors.

\section{Workflow}

The WT workflow in the shot- and angledomains consists of the following steps (see Figure 16):

1) Calculate the shot- or angle-domain crosscorrelation functions using equation 1 or 2 . This step requires the current starting velocity model and the observed data. The migration method can be either RTM or one-way wave-equation migration.

2) Calculate the semblance spectrum. Equation 19 or 20 is used to fit the RTR curve to the shot- or angle-domain crosscorrelation functions.

3) Pick the maximum energy in the semblance spectrum to obtain the curvature parameters and use them to calculate the RTRs with equation 19 or 20 .

4) Calculate the gradient functions using equations 13 and 14 for the shot-domain method or equation 15 for the angle-domain method, and use them to update the velocity model.

\section{DISCUSSION}

Compared with shot-domain WT, angle-domain WT has benefits and limitations in practical applications. Here, we assume that the RTM operator is used to calculate the crosscorrelation function. One limitation is that angle-domain WT is much more computationally expensive than is shot-domain WT. This is because we need 
to compute the subsurface offset-domain crosscorrelation functions over space and time lags. However, if the double-square-root approximation is used to propagate the wavefield, the shot- and angle-domain crosscorrelation functions have almost the same computation cost. One benefit is that angle-domain WT is more robust for complex models than is shot-domain WT. The main reason is that the angle-domain crosscorrelation function can estimate the traveltime residuals more robustly than the shot-domain crosscorrelation function. Angle-domain CIGs have fewer artifacts and are more coherent than shot-domain CIGs in complex structures (Xu et al., 2001; Zhang et al., 2010). Similarly, the angle-domain crosscorrelation function shares those features with angle-domain CIGs. The numerical examples in Figures 5, 6,10 , and 11 show that it is much easier to estimate the traveltime residual with angle-domain crosscorrelation than with shot-domain crosscorrelation. Another benefit is that the multiarrivals can be accounted for in angle-domain WT. In contrast, shot-domain WT takes into account only single arrivals.

Shot-domain crosscorrelation functions will be characterized by several peaks if there are multiple arrivals for a given reflection. Only one of them is picked as a traveltime residual. However, the different pairs of reflections can be separated in the reflection-angle domain, and the traveltime residual for individual reflections can be estimated by the crosscorrelation of traces (a local plane wave) with different reflection angles. Therefore, angle-domain WT can be more accurate than shot-domain WT in complex structures. In summary, shot-domain WT is more practical for simple models and angle-domain WT is more robust for complex models. However, shot-domain WT will be preferable when high-quality angle gathers cannot be computed, such as with sparse 3D data.

The computational cost of the WT largely depends on the number of the image points and the time or space lags chosen for the inversion. More image points are required to improve the resolution of the inverted velocity model. Generally speaking, shot- and angle-domain WT are much more expensive than FWI. This is because the WT method needs to calculate the shot- and angle-domain crosscorrelation functions, which can be computationally expensive. Compared with DSO, shot-domain WT has almost the same cost, whereas angle-domain WT is more expensive. This is because shot-domain crosscorrelation spans over time lags and angle-domain crosscorrelation spans over time and space lags.

One limitation of the WT method is that it is not an automatic method, and manual picking is sometimes required to ensure a robust estimate of
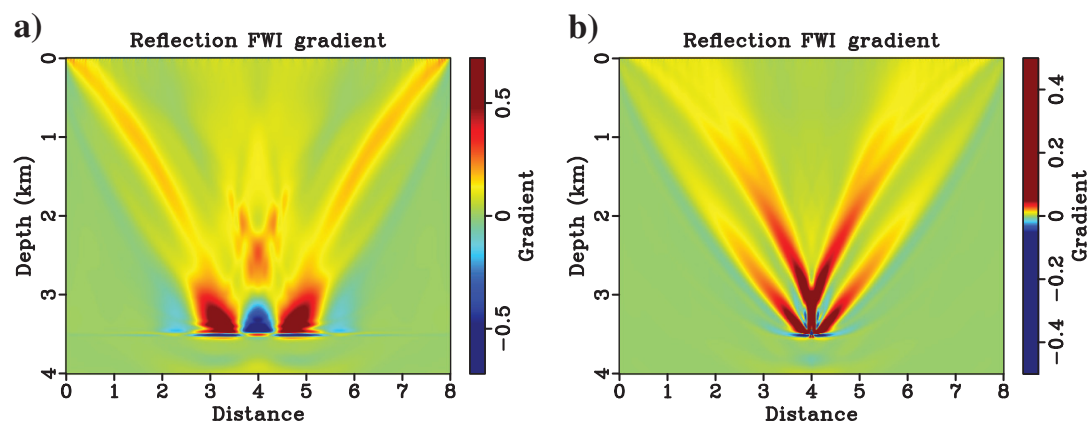

Figure 12. The gradient function of reflection-based FWI using the constant background velocity model $2000 \mathrm{~m} / \mathrm{s}$. Panels (a and b) show the gradient functions calculated for a single point on the reflector.
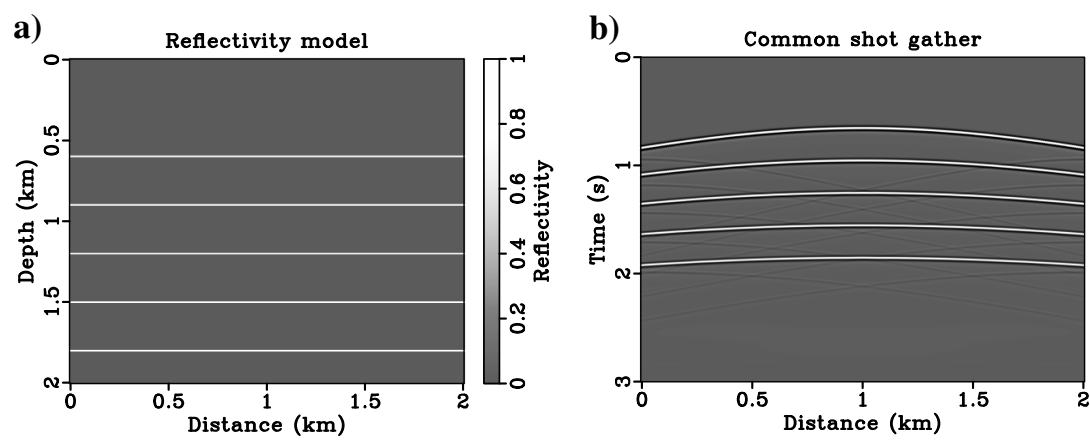

Figure 13. (a) Reflectivity model with the background velocity of $2000 \mathrm{~m} / \mathrm{s}$. (b) A common shot gather with the peak frequency of $20 \mathrm{~Hz}$.
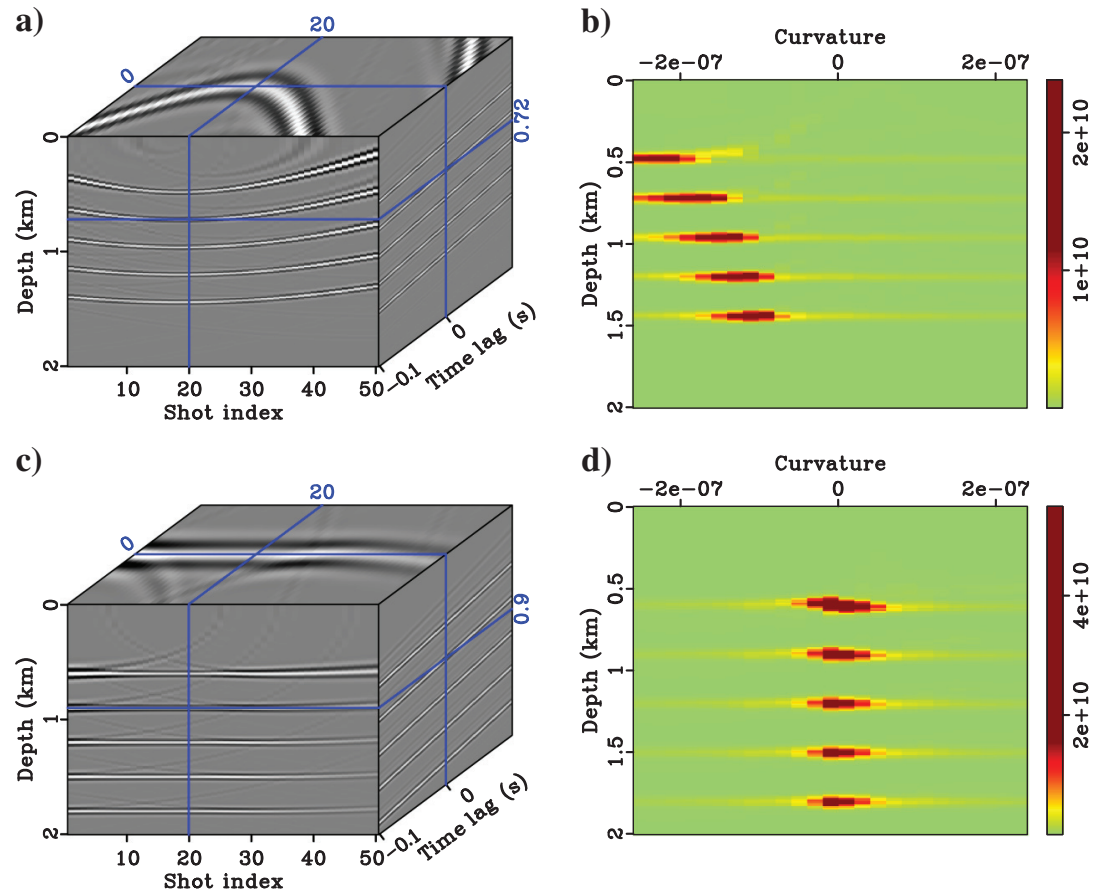

Figure 14. Semblance analysis is used to derive the shot-domain RTRs. (a) Shot-domain crosscorrelation functions generated by RTM for a homogeneous model with a constant velocity of $1600 \mathrm{~m} / \mathrm{s}$. (b) Semblance spectrum, in which energy clusters are picked to find the curvature parameter. Panel (c) is the same as panel (a), and panel (d) is the same as panel (b) except that the true velocity model is used for the computation. 
traveltime residuals. For complex models with short-wavelength errors, semblance analysis based on a single parameter moveout equation does not ensure a precise representation of traveltime residuals.
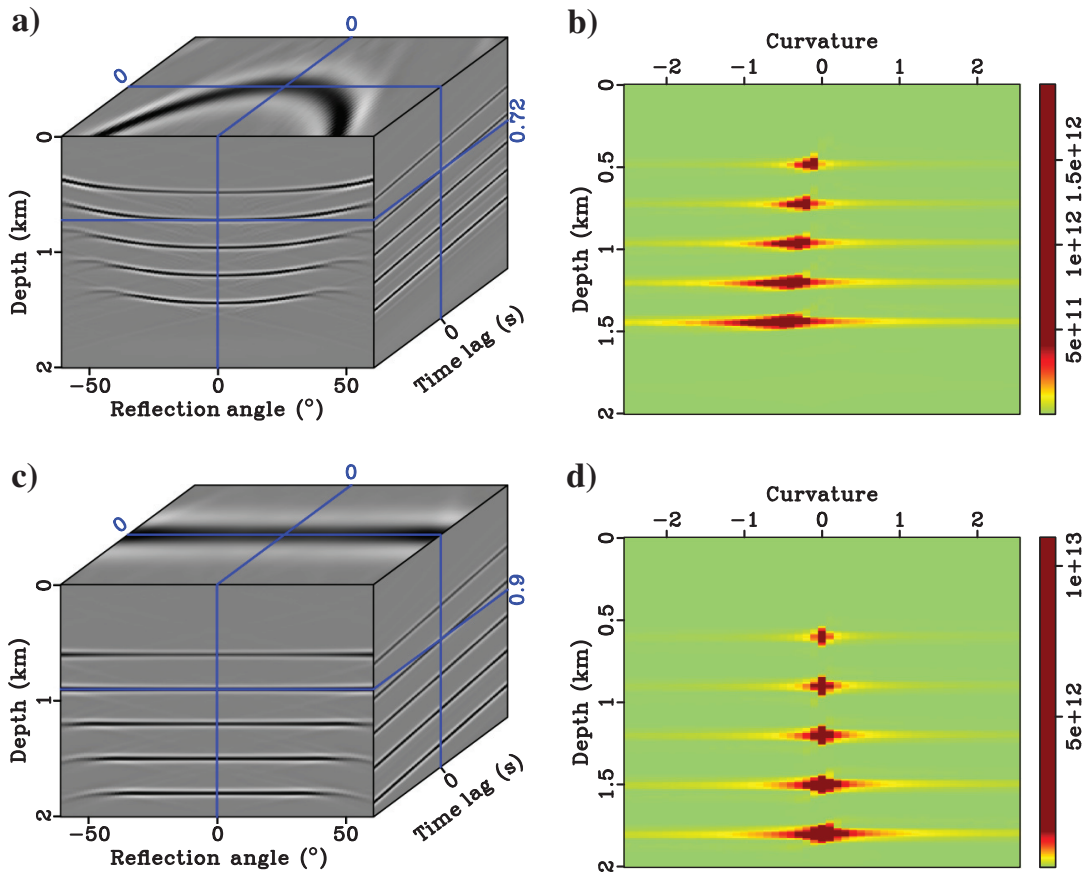

Figure 15. Semblance analysis is used to derive the angle-domain RTRs. (a) Angle-domain crosscorrelation functions generated from a constant velocity of $1600 \mathrm{~m} / \mathrm{s}$ by RTM. (b) Semblance spectra. The energy clusters are picked to find the curvature parameter. Panel (c) is the same as panel (a), and panel (d) is the same as panel (b) except that the true velocity model is used for the computation.

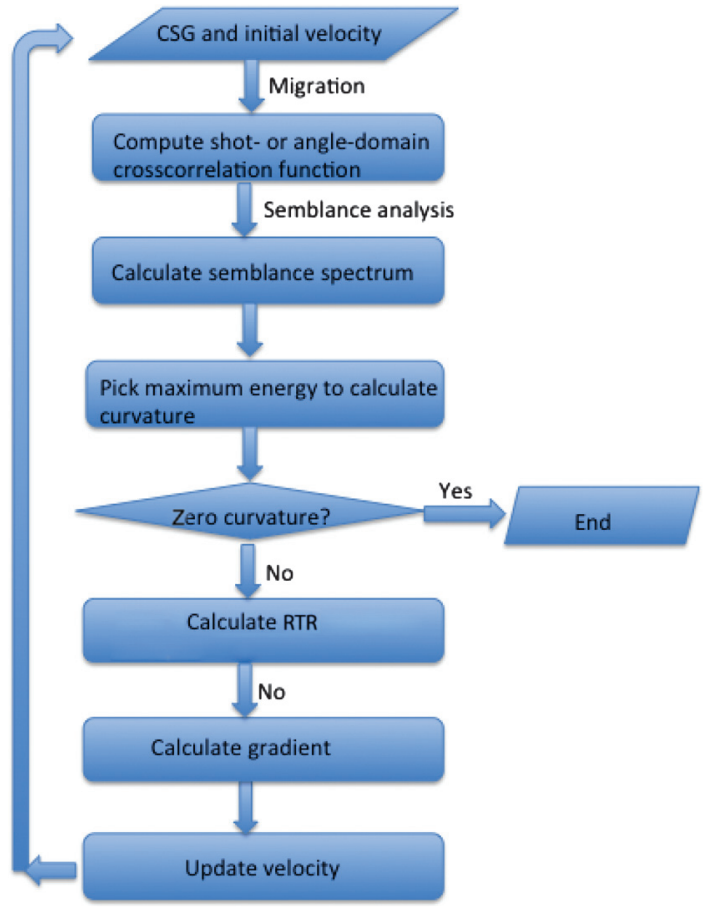

Figure 16. The WT workflow for the shot- and angle-domains.
In this case, multiparameter moveout semblance analysis, predictive painting (Fomel, 2010), and time warping (Burnett and Fomel, 2009) are alternatives for extracting the traveltime residuals.

The WT algorithm might be a useful tool for building complex velocity models, especially for subsalt structures and with strong lateral gradients. Conventional ray-based migration velocity analysis cannot accurately model waves in complex media, and FWI is very sensitive to the initial velocity model. Thus, WT can bridge the gap between these two methods.

\section{CONCLUSIONS}

The WT method is reformulated to estimate the velocity model from reflection traveltimes. No high-frequency assumption is needed, event identification and traveltime picking in data space are unnecessary, the procedure can be adjusted to account for sparse sampling of traces, and there is no need to correctly model amplitudes in the data. Even for complex velocity models, such as subsalt models, the angle-domain crosscorrelation function can sometimes provide a robust estimate of the RTRs for updating the velocity model. This assumes coherent reflections in the redatumed data. The mathematical derivation demonstrates that WT can be interpreted as inverting traveltimes from two virtual-transmission experiments: one for a source at the surface and receivers at the reflector and the other for a source at the reflector and receivers at the recording surface. The resulting crosscorrelation is less prone to cycle skipping than when correlating the predicted and observed traces recorded at the surface. Hence, WT reduces the tendency to get stuck in local minimums. Numerical tests on synthetic data and field recordings are given in the companion paper.

\section{ACKNOWLEDGMENTS}

We acknowledge the 2013 sponsors of the CSIM at KAUST for their support. We also appreciate the KAUST Supercomputing Lab for the use of the Shaheen supercomputer and the Information Technology Department for their hardware support. Some of the computation for this research was accomplished with the Blue Gene/Q supercomputer at IBM in New York. We also acknowledge the support of a KAUST Competitive Research Grant.

\section{APPENDIX A}

\section{DERIVATION OF THE GRADIENT FUNCTION}

Assume the 2D acoustic wave equation

$$
\frac{1}{c^{2}(\mathbf{x})} \frac{\partial^{2} p\left(\mathbf{x}, t \mid \mathbf{x}_{s}\right)}{\partial t^{2}}-\nabla^{2} p\left(\mathbf{x}, t \mid \mathbf{x}_{s}\right)=s\left(t, \mathbf{x}_{s}\right)
$$

where $p\left(\mathbf{x}, t \mid \mathbf{x}_{s}\right)$ denotes the pressure field at time $t$ observed at the location $\mathbf{x}$ due to a source at $\mathbf{x}_{s}, c(\mathbf{x})$ is the velocity model, and 
$s\left(t, \mathbf{x}_{s}\right)$ is the source function. Green's function associated with equation A-1 is denoted as $g\left(\mathbf{x}, t \mid \mathbf{x}_{s}, 0\right)$, and for convenience, we assume no density variations.

The forward-modeled wavefield $p_{s}\left(\mathbf{x}, t \mid x_{s}\right)$ is initiated by the line source at $\mathbf{x}_{s}$

$$
p_{s}\left(\mathbf{x}, t \mid \mathbf{x}_{s}\right)=w(t) * g\left(\mathbf{x}, t \mid \mathbf{x}_{s}, 0\right),
$$

where $w(t)$ is the source wavelet; the symbol * denotes convolution; $p_{g}\left(\mathbf{x}, t \mid \mathbf{x}_{s}\right)$ is the backward-propagated wavefield computed by time-reversed propagation of the observed data $p\left(\mathbf{x}_{g}, t \mid \mathbf{x}_{s}\right)_{\text {obs }}$,

$$
p_{g}\left(\mathbf{x}, t \mid \mathbf{x}_{s}\right)=\int p\left(\mathbf{x}_{g}, t \mid \mathbf{x}_{s}\right)_{\text {obs }} * g\left(\mathbf{x},-t \mid \mathbf{x}_{g}, 0\right) d \mathbf{x}_{g} .
$$

The misfit gradient of shot-domain WT is

$$
\gamma\left(\mathbf{x}^{\prime}\right)=-\frac{\partial S}{\partial c\left(\mathbf{x}^{\prime}\right)}=-\sum_{\mathbf{x}_{s}} \sum_{\mathbf{x}} \Delta \tau \frac{\partial \Delta \tau}{\partial c\left(\mathbf{x}^{\prime}\right)},
$$

where $\gamma\left(\mathbf{x}^{\prime}\right)$ represents the reflection traveltime-misfit gradient at $\mathbf{x}^{\prime}$. Using equations 5 and the rule for an implicit-function derivative, we get

$$
\frac{\partial \Delta \tau}{\partial c\left(\mathbf{x}^{\prime}\right)}=-\frac{\frac{\partial \dot{f}_{\Delta \tau}}{\partial c\left(\mathbf{x}^{\prime}\right)}}{\frac{\partial \dot{f}_{\Delta \tau}}{\partial \Delta \tau}}
$$

where

$$
\frac{\partial \dot{f}_{\Delta \tau}}{\partial \Delta \tau}=\int \ddot{p}_{s}\left(\mathbf{x}, t+\Delta \tau \mid \mathbf{x}_{s}\right) p_{g}\left(\mathbf{x}, t \mid \mathbf{x}_{s}\right) d t,
$$

and

$$
\begin{aligned}
\frac{\partial \dot{f}_{\Delta \tau}}{\partial c\left(\mathbf{x}^{\prime}\right)}= & \int\left[\frac{\partial p_{g}\left(\mathbf{x}, t \mid \mathbf{x}_{s}\right)}{\partial c\left(\mathbf{x}^{\prime}\right)} \dot{p}_{s}\left(\mathbf{x}, t+\Delta \tau \mid \mathbf{x}_{s}\right)\right. \\
& \left.+\frac{\partial \dot{p}_{s}\left(\mathbf{x}, t+\Delta \tau \mid \mathbf{x}_{s}\right)}{\partial c\left(\mathbf{x}^{\prime}\right)} p_{g}\left(\mathbf{x}, t \mid \mathbf{x}_{s}\right)\right] \mathrm{d} t
\end{aligned}
$$

We define $E$ to be $\frac{\partial \dot{f}_{\Delta \tau}}{\partial \Delta \tau}$,

$$
E=\frac{\partial \dot{f}_{\Delta \tau}}{\partial \Delta \tau}
$$

which acts to normalize equation A-7 in equation A-5.

Substituting equations A-5 A-6 and A-7 into equation A-4, we obtain

$$
\gamma\left(\mathbf{x}^{\prime}\right)=\gamma_{1}\left(\mathbf{x}^{\prime}\right)+\gamma_{2}\left(\mathbf{x}^{\prime}\right)
$$

where

$$
\gamma_{1}\left(\mathbf{x}^{\prime}\right)=\sum_{\mathbf{x}_{s}} \sum_{\mathbf{x}} \int \frac{\Delta \tau}{E} \frac{\partial p_{g}\left(\mathbf{x}, t \mid \mathbf{x}_{s}\right)}{\partial c\left(\mathbf{x}^{\prime}\right)} \dot{p}_{s}\left(\mathbf{x}, t+\Delta \tau \mid \mathbf{x}_{s}\right) \mathrm{d} t,
$$

and

$$
\gamma_{2}\left(\mathbf{x}^{\prime}\right)=\sum_{\mathbf{x}_{s}} \sum_{\mathbf{x}} \int \frac{\Delta \tau}{E} \frac{\partial \dot{p}_{s}\left(\mathbf{x}, t+\Delta \tau \mid \mathbf{x}_{s}\right)}{\partial c\left(\mathbf{x}^{\prime}\right)} p_{g}\left(\mathbf{x}, t \mid \mathbf{x}_{s}\right) \mathrm{d} t .
$$

In terms of Born modeling,

$$
\Delta p\left(\mathbf{x}, t \mid \mathbf{x}_{s}\right)=\int \dot{p}\left(\mathbf{x}^{\prime}, t \mid \mathbf{x}_{s}\right) * \dot{g}\left(\mathbf{x}, t \mid \mathbf{x}^{\prime}, 0\right) \frac{2 \Delta c\left(\mathbf{x}^{\prime}\right)}{c\left(\mathbf{x}^{\prime}\right)^{3}} d \mathbf{x}^{\prime},
$$

where $\Delta c\left(\mathbf{x}^{\prime}\right)$ is the velocity perturbation and $\Delta p\left(\mathbf{x}, t \mid \mathbf{x}_{s}\right)$ is the wavefield perturbation. Assuming

$$
\Delta c\left(\mathbf{x}^{\prime}\right)=\Delta c \delta\left(\mathbf{x}-\mathbf{x}^{\prime}\right),
$$

we get the following equation:

$$
\frac{\partial p\left(\mathbf{x}, t \mid \mathbf{x}_{s}\right)}{\partial c\left(\mathbf{x}^{\prime}\right)}=\frac{2}{c\left(\mathbf{x}^{\prime}\right)^{3}} \dot{p}\left(\mathbf{x}^{\prime}, t \mid \mathbf{x}_{s}\right) * \dot{g}\left(\mathbf{x}, t \mid \mathbf{x}^{\prime}, 0\right) .
$$

Equation A-11 can be rewritten as

$$
\begin{aligned}
\gamma_{2}\left(\mathbf{x}^{\prime}\right)= & \frac{2}{c\left(\mathbf{x}^{\prime}\right)^{3}} \sum_{\mathbf{x}_{s}} \sum_{\mathbf{x}} \int \frac{\Delta \tau}{E} \dot{p}_{s}\left(\mathbf{x}^{\prime}, t+\Delta \tau \mid \mathbf{x}_{s}\right) \\
& * \ddot{g}\left(\mathbf{x}, t \mid \mathbf{x}^{\prime}, 0\right) p_{g}\left(\mathbf{x}, t \mid \mathbf{x}_{s}\right) d t .
\end{aligned}
$$

Using the identity (Tarantola, 1987)

$$
\int[f(t) * g(t)] h(t) d t=\int g(t)[f(-t) * h(t)] d t,
$$

we can rewrite equation $\mathrm{A}-15$ as

$$
\begin{array}{r}
\gamma_{2}\left(\mathbf{x}^{\prime}\right)=\frac{2}{c\left(\mathbf{x}^{\prime}\right)^{3}} \sum_{\mathbf{x}_{s}} \sum_{\mathbf{x}} \int \frac{\Delta \tau}{E} \dot{p}_{s}\left(\mathbf{x}^{\prime}, t+\Delta \tau \mid \mathbf{x}_{s}\right) \\
{\left[\ddot{p}_{g}(\mathbf{x}, t) * g\left(\mathbf{x}^{\prime},-t \mid \mathbf{x}, 0\right)\right] d t .}
\end{array}
$$

Similarly, substituting equation A-3 into A-10 gives

$$
\begin{aligned}
\gamma_{1}\left(\mathbf{x}^{\prime}\right)= & \sum_{\mathbf{x}_{s}} \sum_{\mathbf{x}} \int \frac{\Delta \tau}{E} p_{g}\left(\mathbf{x}_{g}, t \mid \mathbf{x}_{s}\right) \\
& * \frac{\partial g\left(\mathbf{x},-t \mid \mathbf{x}_{g}, 0\right)}{\partial c\left(\mathbf{x}^{\prime}\right)} \dot{p}_{s}\left(\mathbf{x}, t+\Delta \tau \mid \mathbf{x}_{s}\right) \mathrm{d} t .
\end{aligned}
$$

Equation A-13 can be rewritten as

$$
\frac{\partial g\left(\mathbf{x},-t \mid \mathbf{x}_{g}, 0\right)}{\partial c\left(\mathbf{x}^{\prime}\right)}=\frac{2}{c\left(\mathbf{x}^{\prime}\right)^{3}} \dot{g}\left(\mathbf{x}^{\prime},-t \mid \mathbf{x}_{g}, 0\right) * \dot{g}\left(\mathbf{x},-t \mid \mathbf{x}^{\prime}, 0\right) \text {. }
$$


Substituting equation A-19 into A-18, we rewrite equation A-18 as

$$
\begin{array}{r}
\gamma_{1}\left(\mathbf{x}^{\prime}\right)=\frac{2}{c\left(\mathbf{x}^{\prime}\right)^{3}} \sum_{\mathbf{x}_{s}} \sum_{\mathbf{x}} \int \frac{\Delta \tau}{E} p_{g}\left(\mathbf{x}_{g}, t \mid \mathbf{x}_{s}\right) \\
* \dot{g}\left(\mathbf{x}^{\prime},-t \mid \mathbf{x}_{g}, 0\right) * \dot{g}\left(\mathbf{x},-t \mid \mathbf{x}^{\prime}, 0\right) \dot{p}_{s}\left(\mathbf{x}, t+\Delta \tau \mid \mathbf{x}_{s}\right) \mathrm{d} t .
\end{array}
$$

Using equation A-16, equation A-20 becomes

$$
\begin{gathered}
\gamma_{1}\left(\mathbf{x}^{\prime}\right)=\frac{2}{c\left(\mathbf{x}^{\prime}\right)^{3}} \sum_{\mathbf{x}_{s}} \sum_{\mathbf{x}} \int\left[\int \frac{\Delta \tau}{E} \dot{p}_{g}\left(\mathbf{x}_{g}, t \mid \mathbf{x}_{s}\right) * g\left(\mathbf{x}^{\prime},-t \mid \mathbf{x}_{g}, 0\right) d \mathbf{x}_{g}\right] \\
{\left[\ddot{p}_{s}\left(\mathbf{x}, t+\Delta \tau \mid \mathbf{x}_{s}\right) * g\left(\mathbf{x}^{\prime}, t \mid \mathbf{x}, 0\right)\right] \mathrm{d} t .}
\end{gathered}
$$

In summary, the misfit gradient (equation A-9) can be written as

$$
\gamma\left(\mathbf{x}^{\prime}\right)=\gamma_{1}\left(\mathbf{x}^{\prime}\right)+\gamma_{2}\left(\mathbf{x}^{\prime}\right)
$$

where

$$
\gamma_{1}\left(\mathbf{x}^{\prime}\right)=\frac{2}{c\left(\mathbf{x}^{\prime}\right)^{3}} \sum_{\mathbf{x}_{s}} \sum_{\mathbf{x}} \int \overbrace{\left[\int \frac{\Delta \tau}{E} \dot{p}_{g}\left(\mathbf{x}_{g}, t \mid \mathbf{x}_{s}\right) * g\left(\mathbf{x}^{\prime},-t \mid \mathbf{x}_{g}, 0\right) d \mathbf{x}_{g}\right]}^{\text {back propagation of the observed data }}
$$

forward propagation of the redatumed source ${ }_{\mathrm{d}}$

$$
\overbrace{\left[\ddot{p}_{s}\left(\mathbf{x}, t+\Delta \tau \mid \mathbf{x}_{s}\right) * g\left(\mathbf{x}^{\prime}, t \mid \mathbf{x}, 0\right)\right]}
$$

and

$$
\gamma_{2}\left(\mathbf{x}^{\prime}\right)=\frac{2}{c\left(\mathbf{x}^{\prime}\right)^{3}} \sum_{\mathbf{x}_{s}} \sum_{\mathbf{x}} \int \overbrace{\left[\frac{\Delta \tau}{E} \ddot{p}_{g}\left(\mathbf{x}, t \mid \mathbf{x}_{s}\right) * g\left(\mathbf{x}^{\prime},-t \mid \mathbf{x}, 0\right)\right]}^{\text {back propagation of the redatumed data }}
$$

forward propagation of the source $\mathrm{d} t$.

$$
\overbrace{\left[\dot{p}_{s}\left(\mathbf{x}^{\prime}, t+\Delta \tau \mid \mathbf{x}_{s}\right)\right]}
$$

Equations A-23 and A-24 are the gradients for the shot-domain WT method.

The angle-domain misfit gradient is

$$
\gamma\left(\mathbf{x}^{\prime}\right)=-\frac{\partial S}{\partial c\left(\mathbf{x}^{\prime}\right)}=-\sum_{\mathbf{x}} \sum_{\theta} \Delta \tau \frac{\partial \Delta \tau}{\partial c\left(\mathbf{x}^{\prime}\right)}
$$

where $\gamma\left(\mathbf{x}^{\prime}\right)$ represents the reflection traveltime-misfit gradient. Using the chain rule for an implicit function derivative, we get

$$
\frac{\partial \Delta \tau}{\partial c\left(\mathbf{x}^{\prime}\right)}=-\frac{\frac{\partial \dot{f}_{\Delta \tau}}{\partial c\left(\mathbf{x}^{\prime}\right)}}{\frac{\partial \dot{f}_{\Delta \tau}}{\partial \Delta \tau}}
$$

where

$$
\begin{gathered}
\frac{\partial \dot{f}_{\Delta \tau}}{\partial \Delta \tau}=\int d \mathbf{x}_{s} \int d h \int \ddot{p}_{s}\left(\mathbf{x}-h, z+h \tan \theta, t+\Delta \tau \mid \mathbf{x}_{s}\right) \\
p_{g}\left(\mathbf{x}+h, z+h \tan \theta, t \mid \mathbf{x}_{s}\right) \mathrm{d} t
\end{gathered}
$$

and

$$
\begin{gathered}
\frac{\partial \dot{f}_{\Delta \tau}}{\partial c\left(\mathbf{x}^{\prime}\right)}=\int d \mathbf{x}_{s} \int d h \int\left[\frac{\partial p_{g}\left(\mathbf{x}+h, z+h \tan \theta, t \mid \mathbf{x}_{s}\right)}{\partial c\left(\mathbf{x}^{\prime}\right)}\right. \\
\dot{p}_{s}\left(\mathbf{x}-h, z+h \tan \theta, t+\Delta \tau \mid \mathbf{x}_{s}\right) \\
+\frac{\partial \dot{p}_{s}\left(\mathbf{x}-h, z+h \tan \theta, t+\Delta \tau \mid \mathbf{x}_{s}\right)}{\partial c\left(\mathbf{x}^{\prime}\right)} \\
\left.p_{g}\left(\mathbf{x}+h, z+h \tan \theta, t \mid \mathbf{x}_{s}\right)\right] \mathrm{d} t .
\end{gathered}
$$

Under the Born approximation, we can rewrite the misfit gradient A-25 as

$$
\begin{aligned}
& \gamma\left(\mathbf{x}^{\prime}\right)=\frac{2}{c\left(\mathbf{x}^{\prime}\right)^{3}} \sum_{\theta} \sum_{\mathbf{x}} \frac{\Delta \tau(\mathbf{x}, \theta)}{E} \int d \mathbf{x}_{s} \int d h \int \dot{p}_{g}\left(\mathbf{x}^{\prime}, t \mid \mathbf{x}_{s}\right) \\
& {\left[\ddot{p}_{s}\left(\mathbf{x}-h, z+h \tan \theta, t+\Delta \tau \mid \mathbf{x}_{s}\right) * g\left(\mathbf{x}^{\prime}, t \mid \mathbf{x}+h, z+h \tan \theta, 0\right)\right] d t} \\
& +\frac{2}{c\left(\mathbf{x}^{\prime}\right)^{3}} \sum_{\theta} \sum_{\mathbf{x}} \frac{\Delta \tau(\mathbf{x}, \theta)}{E} \int d \mathbf{x}_{s} \int d h \int \dot{p}_{s}\left(\mathbf{x}^{\prime}, t+\Delta \tau \mid \mathbf{x}_{s}\right) \\
& {\left[\ddot{p}_{g}\left(\mathbf{x}+h, z+h \tan \theta, t \mid \mathbf{x}_{s}\right) * g\left(\mathbf{x}^{\prime},-t \mid \mathbf{x}-h, z+h \tan \theta, 0\right)\right] \mathrm{d} t .}
\end{aligned}
$$

Equation A-29 has a physical interpretation similar to that of equations A-23 and A-24. The difference is that the backward-propagated wave is a local plane wave with a time shift associated with a certain reflection angle.

\section{REFERENCES}

Almomin, A., 2011, Correlation-based wave-equation migration velocity analysis: 81st Annual International Meeting, SEG, Expanded Abstracts, 3891-3887.

Al-Saleh, S., and J. Jiao, 2012, Migration velocity analysis using traveltime wavefield tomography: Geophysics, 77, no. 5, U73-U85, doi: 10.1190/ geo2011-0440.1.

Biondi, B., and W. Symes, 2004, Angle-domain common-image gathers for migration velocity analysis by wavefield-continuation imaging: Geophysics, 69, 1283-1298, doi: 10.1190/1.1801945.

Bunks, C., F. Saleck, S. Zaleski, and G. Chavent, 1995, Multiscale seismic waveform inversion: Geophysics, 60, 1457-1473, doi: 10.1190/1.1443880.

Burnett, W., and S. Fomel, 2009, Moveout analysis by time-warping: 79th Annual International Meeting, SEG, Expanded Abstracts, 3710-3714.

Crase, E., C. Wideman, M. Noble, and A. Tarantola, 1992, Nonlinear elastic waveform inversion of land seismic reflection data: Journal of Geophysical Research, 97, 4685-4704, doi: 10.1029/90JB00832.

Fomel, S., 2004, Theory of 3-D angle gathers in wave-equation seismic imaging: 72nd Annual International Meeting, SEG, Expanded Abstracts, 1053-1056.

Fomel, S., 2010, Predictive painting of 3D seismic volumes: Geophysics, 75, no. 4, A25-A30, doi: 10.1190/1.3453847.

Luo, S., and D. Hale, 2013, Unfaulting and unfolding 3D seismic images: Geophysics, no. 4, 78, O45-O56, doi: 10.1190/geo2012-0350.1.

Luo, Y., and G. Schuster, 1991a, Wave equation traveltime inversion: Geophysics, 56, 645-653, doi: 10.1190/1.1443081.

Luo, Y., and G. Schuster, 1991b, Wave equation inversion of skeletonized geophysical data: Geophysical Journal International, 105, 289-294, doi: 10.1111/j.1365-246X.1991.tb06713.x

Ma, Y., and D. Hale, 2013, Wave-equation reflection traveltime inversion with dynamic warping and full-waveform inversion: Geophysics, 78, no. 6, R223-R233, doi: 10.1190/geo2013-0004.1.

Mora, P., 1987, Nonlinear two-dimensional elastic inversion of seismic data: Geophysics, 52, 1211-1228, doi: 10.1190/1.1442384.

Mora, P., 1989, Inversion = migration + tomography: Geophysics, 54, 15751586, doi: $10.1190 / 1.1442625$. 
Plessix, R. E. G. Baeten, J. W. de Maag, M. Klaassen, Z. Rujie, and T. Zhifei, 2010, Application of acoustic full waveform inversion to a low frequency large-offset land data set: 78th Annual International Meeting, SEG, Expanded Abstracts, 930-934.

Plessix, R. E., and H. Rynja, 2010, VTI full waveform inversion: A parameterization study with a narrow azimuth streamer data example: 78th Annual International Meeting, SEG, Expanded Abstracts, 962-966.

Pratt, R. G., C. Shin, and G. J. Hicks, 1998, Gauss-Newton and full Newton methods in frequency domain seismic waveform inversion: Geophysical Journal International, 133, 341-362, doi: 10.1046/j.1365-246X.1998 .00498.x.

Sava, P., and B. Biondi, 2004a, Wave-equation migration velocity analysis. I. Theory: Geophysical Prospecting, 52, 593-606, doi: 10.1111/j.13652478.2004.00447.x.

Sava, P., and B. Biondi, 2004b, Wave-equation migration velocity analysis II. Examples: Geophysical Prospecting, 52, 607-623, doi: 10.1111/j $.1365-2478.2004 .00448 . x$

Sava, P., and S. Fomel, 2003, Angle-domain common-image gathers by wavefield continuation methods: Geophysics, 68, 1065-1074, doi: 10 $.1190 / 1.1581078$.

Shen, P., and H. Calandra, 2005, One-way waveform inversion within the framework of adjoint state differential migration: 75th Annual International Meeting, SEG, Expanded Abstracts, 1709-1712.

Shen, P., and W. Symes, 2008, Automatic velocity analysis via shot profile migration: Geophysics, 73, no. 5, VE49-VE59, doi: 10.1190/1.2972021.

Shen, P., W. Symes, and C. Stolk, 2003, Differential semblance velocity analysis by wave-equation migration: 73rd Annual International Meeting, SEG, Expanded Abstracts, 2132-2135.

Sirgue, L., and R. G. Pratt, 2004, Efficient waveform inversion and imaging: A strategy for selecting temporal frequencies: Geophysics, 69, 231-248, doi: $10.1190 / 1.1649391$

Symes, W., and M. Kern, 1994, Inversion of reflection seismograms by differential semblance analysis: Algorithm structure and synthetic examples: Geophysical Prospecting, 42, 565-614, doi: 10.1111/j.1365-2478.1994 .tb00231.x.

Tang, Y., S. Lee, A. Baumstein, and D. Hinkley, 2013, Tomographically enhanced full wavefield inversion: 83rd Annual International Meeting, SEG, Expanded Abstracts, 1037-1041.

Tarantola, A., 1986, A strategy for nonlinear elastic inversion of seismic reflection data: Geophysics, 51, 1893-1903, doi: 10.1190/1.1442046.

Tarantola, A., 1987, Inverse problem theory: Elsevier.

Virieux, J., and S. Operto, 2009, An overview of full-waveform inversion in exploration geophysics: Geophysics, 74, no. 6, WCC1-WCC26, doi: 10 $.1190 / 1.3238367$.

Wang, B., C. Mason, M. Guo, K. Yoon, J. Cai, J. Ji, and Z. Li, 2009, Subsalt velocity update and composite imaging using reverse-time-migration based delayed-imaging-time scan: Geophysics, 74, no. 6, WCA159WCA166, doi: 10.1190/1.3227152.

Wang, S., F. Chen, H. Zhang, and Y. Shen, 2013, Reflection-based full waveform inversion (RFWI) in the frequency domain: 83rd Annual International Meeting, SEG, Expanded Abstracts, 877-881.

Woodward, M., 1992, Wave-equation tomography: Geophysics, 57, 15-26, doi: $10.1190 / 1.1443179$.

Xu, S., H. Chauris, G. Lambare, and M. Noble, 2001, Common-angle migration: A strategy for imaging complex media: Geophysics, 66, $1877-$ 1894, doi: 10.1190/1.1487131.

Xu, S., F. Chen, G. Lambare, and Y. Zhang, 2013, Full waveform inversion of reflected seismic data: Journal of Seismic Exploration, 22, 449-462.

Xu, S., Y. Zhang, and B. Tang, 2011, 3D angle gathers from reverse time migration: Geophysics, 76, no. 2, S77-S92, doi: 10.1190/1.3536527.

Yang, T., and P. Sava, 2011, Wave-equation migration velocity analysis with time-lag imaging: Geophysical Prospecting, 59, 635-650, doi: 10.1111/ .1365-2478.2011.00954.x.

Yang, T., and P. Sava, 2013, 3D image-domain wavefield tomography using time-lag extended images: 83rd Annual International Meeting, SEG, Expanded Abstracts, 4816-4821.

Yang, T., J. Shragge, and P. Sava, 2013, Illumination compensation for image-domain wavefield tomography: Geophysics, 78, no. 5, U65-U76, doi: $10.1190 /$ geo2012-0278.1.

Zhang, S., Y. Luo, and G. Schuster, 2015, Shot- and angle-domain waveequation traveltime inversion of reflection data: Synthetic and field examples: Geophysics, 80, this issue, doi: 10.1190/geo2014-0223.1.

Zhang, S., G. Schuster, and Y. Luo, 2011, Wave-equation reflection traveltime inversion: 81st Annual International Meeting, SEG, Expanded Abstracts, 2705-2710.

Zhang, S., G. Schuster, and Y. Luo, 2012, Angle-domain migration velocity analysis using wave-equation reflection traveltime inversion: 82nd Annual International Meeting, SEG, Expanded Abstracts, doi: 10.1190/ segam2012-1123.1.

Zhang, Y., and B. Biondi, 2013, Moveout-based wave-equation migration velocity analysis: Geophysics, 78, no. 2, U31-U39, doi: 10.1190/ geo2012-0082.1.

Zhang, Y., S. Xu, B. Tang, B. Bai, Y. Huang, and T. Huang, 2010, Angle gathers from reverse time migration: The Leading Edge, 29, 1364-1371, doi: $10.1190 / 1.3517308$

Zhou, C., W. Cai, Y. Luo, G. Schuster, and S. Hassanzadeh, 1995, Acoustic wave-equation traveltime and waveform inversion of crosshole seismic data: Geophysics, 60, 765-773, doi: 10.1190/1.1443815.

Zhou, H., A. Lasse, and G. Zhang, 2012, Fundamental issues in full waveform inversion: 82nd Annual International Meeting, SEG, Expanded Abstracts, doi: 10.1190/segam2012-0878.1. 